\title{
A clinical trial using co-trimoxazole in an attempt to reduce wound infection rates in dog bite wounds
}

\author{
David Andrew Jones* and Thomas Nigel Stanbridge
}

Accident and Emergency Department, Wythenshawe Hospital, Manchester, UK.

\begin{abstract}
Summary: One hundred and thirteen patients were entered into a randomized, prospective doubleblind, placebo controlled trial to assess the use of co-trimoxazole in reducing wound infections after dog bites. Although there was a reduction in the wound infection rate from $13.8 \%$ in the placebo group to $5.5 \%$ in the treatment group this did not reach statistical significance $(P=0.135)$. If hand wounds are considered separately, no infections occurred in the treatment group and a benefit seems likely.
\end{abstract}

\section{Introduction}

Dog bite wounds are a common problem seen in accident and emergency departments. Prevention of tetanus and rabies where appropriate, together with adequate cleansing of such wounds, are universally accepted measures. The prophylactic use of antibiotics in an attempt to reduce wound infections is controversial. Previous studies have failed to show any conclusive benefit from the routine use of antibiotics in such wounds (Callaham, 1980; Elenbaas et al., 1981).

Recent review articles in the British Medical Journal (Snook, 1982) and the Lancet (Anon, 1983) have advocated their use in certain wounds, while accepting that further information was required. This prospective, randomized, double-blind placebo controlled trial was undertaken to further evaluate the routine use of antibiotics in patients with dog bite wounds.

\section{Patients and methods}

Patients treated in the accident and emergency department for dog bite wounds were randomly allocated to receive either a $5 \mathrm{~d}$ course of co-trimoxazole $960 \mathrm{mg}$ twice daily, or a placebo. Children less than 12 y of age took half the dose. Patients with superficial abrasions, those having other medical problems requiring antibiotic treatment or allergies to co-trimoxazole, and children less than 3 y of age were excluded. Details of the patient's age and sex and of the wound site (hand, face or elsewhere), type (puncture or lacera-

\footnotetext{
* Correspondence and present address: D.A. Jones, F.R.C.S., Department of Clinical Studies, Park Hospital, Davyhulme, Manchester M31 3SL, UK.

Accepted: 27 February 1985
}

tion) and age at presentation were recorded.

Wounds were re-assessed 1 week later and those considered to be infected had a swab sent for bacterial culture. Statistical analysis of results was by the $\mathrm{Chi}^{2}$ test and Fisher's exact test. The trial was approved by the Hospital Ethical Committee.

\section{Results}

One hundred and thirteen patients were included in the study. Among those excluded was 1 patient who already had a digital volar space infection resulting from a dog bite $2 \mathrm{~d}$ before presentation and in whom antibiotics clearly could not be withheld. Fifty eight received placebo and 55 received co-trimoxazole. The groups were well matched in terms of age and sex, and site, type and age of wound.

Thirty five patients failed to attend for the 1 week follow up and these wounds have been included in the non-infected group because it is reasonable to suppose that they would have returned had infection ensued. There were fewer wound infections in the co-trimoxazole treated patients than in those given placebo, but this difference failed to achieve statistical significance (see Table I). When wounds at different sites were assessed separately, those at sites other than the hand are seen to follow the same pattern as the whole group. With hand wounds, however, there was a significantly higher proportion of lacerations and the reduced number of wound infections seen in the co-trimoxazole treated patients reaches marginal statistical significance (Table II).

Eleven patients developed clinically diagnosed wound infection, 3 in the co-trimoxazole treated group 
Table I Incidence of wound infections

\begin{tabular}{lccc}
\hline & Total number & Non-infected & Infected \\
\hline Placebo & $58(100)$ & $50(86.2)$ & $8(13.8)$ \\
Co-trimoxazole & $55(100)$ & $52(94.5)$ & $3(5.5)^{*}$ \\
\hline
\end{tabular}

Figures in parentheses are \%.

$95 \%$ confidence limits for the proportion infected were $6.5 \%-25.4 \%$ for the placebo group and $1.1 \%-15.1 \%$ for the treated group.

$* P=0.135$

and 8 in the placebo group. Of the 3 in the cotrimoxazole treated group, Escherichia coli was isolated from one, no pathogen was isolated from a second, and culture was not performed in the third. In the placebo treated group there were 4 Staphylococcus aureus infections, 1 mixed infection with Proteus and Bacteroides, in 2 no pathogens were cultured and in 1 no specimen was taken.

\section{Discussion}

Dog bite wounds are common, but a large percentage are minor, supeficial abrasions. There is a small but definite morbidity and mortality associated with infection in the more serious lacerated and puncture wounds (Fiala et al., 1977; Jarvis et al., 1981).

Previous reports (Callaham, 1980; Snook, 1983) have considered patients aged over $50 \mathrm{y}$, those with puncture wounds and hand wounds, and those delaying presentation to medical attention for over $24 \mathrm{~h}$ to be at high risk. We chose to use co-trimoxazole for our study because most of the organisms causing infection in dog bite wounds are sensitive to it (Snook, 1982) and it is relatively inexpensive and commonly available.

The infection rate of $13.8 \%$ seen in our placebo treated group concurs with a range of $6 \%$ to $13 \%$ noted in other studies (Callaham, 1978; Aghababian \& Conte, 1980).

The difference in wound infection rates between treatment and placebo groups failed to reach statis-

\section{References}

AGHABABIAN, R.V. \& CONTE, J.E. (1980). Mammalian bite wounds. Annals of Emergency Medicine, 9, 79.

ANON (1983). Animal bites and infection. Lancet, ii, 553.

CALlAHAM, M. (1978). Treatment of common dog bites: infection risk factors. Journal of the American College of Emergency Physicians, 7, 83.

CALlaHAM, M. (1980). Dog bite wounds. Journal of the American Medical Association, 244, 2327.

ELENBAAS, R.M., MCNABNEY, W.K. \& ROBINSON, W.A. (1981). Prophylactic antibiotics and dog bite wounds.
Table II Infection in hand wounds

\begin{tabular}{lcll}
\hline & Total number & Non-infected & Infected \\
\hline Placebo & $24(100)$ & $20(83.3)$ & $4(16.7)$ \\
Co-trimoxazole & $23(100)$ & $23(100)$ & $0(0)^{*}$ \\
\hline
\end{tabular}

Figures in parentheses are $\%$.

$95 \%$ confidence limits for the proportion infected were $4.7 \%-37.4 \%$ for the placebo group and $0-14.8 \%$ for the treated group.

* Fisher's exact test $P=0.0595$.

tical significance $(P=0.135)$. The probability of a type 2 error was estimated at 0.65 , making the result inconclusive, as it would be inappropriate to interpret non-significance as implying no difference between the groups. Three hundred and seventy patients in each group would be required to test the observed difference with a probability of type 2 error of $10 \%$.

In the sub-group of patients with hand wounds the difference in infection rates reached marginal significance (Fisher's exact test $P=0.06$ ). The probability of a type 2 error was estimated at 0.55 . It seems likely that there is therefore a real difference between the two groups. The higher incidence of lacerations in these patients may possibly be related to the thinner skin on the dorsum of the hand and its likelihood of tearing when the victim withdraws it from attack.

We failed to grow pathogenic organisms from 3 infected wounds but we feel that clinical evidence of infection is a more reliable indicator. Any wound surface contains bacteria and their mere presence on culture is no proof of infection.

In conclusion, we feel that the routine treatment of dog bite wounds with antibiotics is not justified, but that hand wounds should be considered for such treatment.

\section{Acknowledgement}

We would like to thank Mr Chris Roberts MSc., Department of Medical Statistics, University Hospital of South Manchester, for statistical help.

Journal of the American Medical Association, 246, 833.

FIALA, M., BAUER, H. KHALEELI, M. \& GIORGIO, A. (1977). Dog bite bacteroides infection, coagulopathy, renal microangiopathy. Annals of Internal Medicine, 87, 248.

JARVIS, W.R., BANKO, S., SNYDER, E. \& BALTIMORE, R.S. (1981). Pasteurella multocida osteomyelitis following dog bites. American Journal of Diseases of Children, 135, 625. SNOOK, R. (1982). Dog bites man. British Medical Journal, 284, 293. 